\title{
AVALIAÇÃO DE MAPAS CONCEITUAIS ELABORADOS POR ESTUDANTES DE ENFERMAGEM COM O APOIO DE SOFTWARE
}

\author{
Ana Luísa Petersen Cogoㅁ, Eva Néri Rubim Pedro², Ana Paula Scheffer Schell da Silva, Andréia \\ Martins Specht ${ }^{4}$
}

\begin{abstract}
${ }^{1}$ Doutoranda do Programa de Pós-Graduação em Enfermagem (PPGENF) da Universidade Federal do Rio Grande do Sul (UFRGS). Professor Assistente da Escola de Enfermagem (EE) da UFRGS. Rio Grande do Sul, Brasil. E-mail: analuisa@ enf.ufrgs.br

${ }^{2}$ Doutora em Educação. Professor Associado da EE/UFRGS. Rio Grande do Sul, Brasil. E-mail: evapedro@enf.ufrgs.br

${ }^{3}$ Doutoranda do PPGENF/UFRGS. Professor Assistente do Curso de Enfermagem da Universidade Federal do Pampa. Rio Grande do Sul, Brasil. E-mail: anaschell@gmail.com

${ }^{4}$ Aluna do Curso de Graduação da EE/UFRGS. Bolsista de Iniciação Científica da Pró-Reitoria de Pesquisa da UFRGS. Rio Grande do Sul, Brasil. E-mail: deiaspecht@yahoo.com.br
\end{abstract}

RESUMO: Este estudo objetiva avaliar a estratégia de utilização de mapas conceituais elaborados com o software CmapTools ${ }^{\circledR}$, no curso na modalidade a distância denominado Introdução a Anamnese e ao Exame Físico de Enfermagem. Trata-se de um estudo de caso qualitativo, que contou com a participação de 20 estudantes de graduação em enfermagem. Os dados foram obtidos por meio da avaliação dos 11 mapas conceituais elaborados no curso, da avaliação escrita da atividade realizada e das informações obtidas pela técnica de grupo focal. A partir da análise temática, identificou-se que os estudantes demonstraram apropriação dos principais conceitos necessários na elaboração dos mapas conceituais, devendo ainda aperfeiçoar a expressão de reflexões críticas. Os mesmos avaliaram a atividade como positiva por possibilitar a síntese dos temas em estudo e ter aplicações futuras. Constatou-se a importância de desenvolver, com os estudantes de enfermagem, mapas conceituais com o apoio de software.

DESCRITORES: Enfermagem. Educação em enfermagem. Educação à distância. Tecnologia educacional. Aprendizagem.

\section{EVALUATION OF CONCEPT MAPS DEVELOPED BY NURSING STUDENTS WITH SOFTWARE SUPPORT}

\begin{abstract}
This study aimed at evaluating the strategy of using conceptual maps created with the CmapTools® software in "Introduction to Anamnesis and Physical Exams in Nursing", a distance-learning course. Twenty individuals participated in this qualitative case study. Data was obtained by means of evaluating 11 conceptual maps created by the students in the course, submitting them to descriptive statistics; through student written evaluations of the activity; and the information obtained by the focus group technique, both submitted to thematic analysis. This study identified that the students demonstrated an approximation to the main concepts needed in map creation, with a need to improve their critical thinking expression. The students evaluated the activity positively, in that it allows for synthesis of the themes being studied and has future applications. Creating conceptual maps together with nursing students by using software support was considered important.
\end{abstract}

DESCRIPTORS: Nursing. Education, nursing. Education, distance. Educational technology. Learning.

\section{EVALUACIÓN DE MAPAS CONCEPTUALES ELABORADOS POR ESTUDIANTES DE ENFERMERÍA CON EL APOYO DE SOFTWARE}

RESUMEN: El objetivo del presente estudio es evaluar la estrategia de utilización de mapas conceptuales elaborados con el software CmapTools ${ }^{\circledR}$, en el curso a distancia Introducción a la Anamnesis y al Examen Físico de Enfermería. La investigación, desarrollada con la metodología del estudio de caso cualitativo, contó con la participación de 20 estudiantes de enfermería. Los datos se originaron de la evaluación de los 11 mapas conceptuales elaborados por los alumnos del curso y analizados por medio de la técnica de los grupos focales. A partir del análisis temático se identificó que los alumnos demostraron apropiación de los principales conceptos necesarios para la elaboración de los mapas conceptuales, pero siendo necesario perfeccionar la expresión de reflexiones críticas. Los estudiantes evaluaron positivamente la actividad ya que posibilita la síntesis de los temas estudiados y permite aplicaciones futuras. Se pudo constatar la importancia de desarrollar, con los estudiantes de enfermería, mapas conceptuales utilizando software.

DESCRIPTORES: Enfermería. Educación en enfermería. Educación a distancia. Tecnología educacional. Aprendizaje. 


\section{INTRODUÇÃO}

Nos últimos anos, a utilização de Mapas Conceituais (MCs) tem se destacado no ensino de enfermagem, especialmente com estudantes de cursos de graduação e pós-graduação, por meio de uma proposta pedagógica construtivista. Esses constituem-se em representações gráficas de relações entre conceitos ou de seus significados que estão conectados entre si por verbos. ${ }^{1}$

Os estudos realizados sobre a utilização de MCs no ensino de enfermagem apontam que a metodologia tem sido adotada em diversas disciplinas com eficácia no desenvolvimento do pensamento crítico de estudantes de enfermagem, ${ }^{1-7}$ na organização das informações, ${ }^{5,8-9}$ na compreensão de relações complexas, ${ }^{2,4,10-11}$ na integração da teoria com a prática, ${ }^{12}$ além de ser um recurso de acompanhamento da aprendizagem em cursos online. ${ }^{6}$ Os estudantes, com a utilização dessa ferramenta, são capazes de agregar conhecimentos essenciais, ${ }^{13}$ experiências e soluções que suportam atividades práticas por meio da resolução de problemas, ${ }^{1}$ além de relacionarem os conteúdos das diferentes disciplinas. ${ }^{8}$

A utilização de MCs no ensino do Processo de Enfermagem tem proporcionado a reflexão dos estudantes sobre a arte de cuidar, quando esses são desafiados a estabelecerem relações não-lineares entre suas diferentes etapas. ${ }^{2} \mathrm{O}$ trabalho em grupo constitui-se em uma estratégia para o desenvolvimento de MC que promove a solução de problemas com a negociação coletiva entre os pares. ${ }^{3}$

A avaliação das atividades de desenvolvimento de MCs por estudantes de graduação e de pós-graduação revelou que essas, ao serem realizadas em grupos, foram eficazes no processo de ensino-aprendizagem, e que os alunos mostram satisfação na sua confecção. ${ }^{12-13}$

Um estudo comparou o processo de aprendizagem de estudantes de enfermagem que desenvolveram MCs com outros que tiveram atividades de aula convencional, demonstrando que o MC é uma intervenção eficaz para promover a metacognição. ${ }^{11}$ Isso significa que os estudantes atingiram, após atividades de realização de $\mathrm{MC}$, níveis de discussão mais aprofundados.

Outro estudo com estudantes de graduação em enfermagem, em disciplina sobre saúde e adoecimento, apontou que os MCs sintetizaram os conceitos que eram importantes na aprendizagem e, em comparação com o ensino tradicional, mostrou-se mais eficiente para avaliação dos conhecimentos adquiridos, apesar da necessidade de estudos que confirmem a eficácia desse recurso pedagógico. ${ }^{9,14}$

Os MCs podem ser elaborados em papel, mas hoje conta-se com o auxílio de softwares para sua confecção como, por exemplo, o CmapTools ${ }^{15}{ }^{15}$ Esse software foi selecionado por ser gratuito (http://cmap.ihmc.us/download/), possuir versão na língua portuguesa e ser de fácil manuseio na construção de apresentações gráficas. O MC desenvolvido com apoio de software é utilizado em cursos na modalidade a distância como um facilitador da comunicação professor-aluno, além de ser uma forma de acompanhamento da trajetória de aprendizagem. ${ }^{10}$

A partir das diferentes possibilidades relatadas anteriormente, constata-se que o MC configura-se como uma estratégia de ensino promotora da construção de um conhecimento complexo que integra as partes ao todo e suas múltiplas implicações. A reforma no pensamento, que preconiza o desenvolvimento do conhecimento de uma forma complexa e de análise do contexto, é uma proposta de mudança nas relações de ensino tradicional e conteudista ainda presentes no ensino de enfermagem. ${ }^{16}$

A Escola de Enfermagem da Universidade Federal do Rio Grande do Sul, em curso de extensão no ano de 2008, utilizou MCs para o acompanhamento e avaliação de alunos na questão da Sistematização da Assistência de Enfermagem, especialmente, na anamnese e no exame físico, através da leitura de referenciais teóricos e discussões sobre o tema. Dessa forma, o objetivo do presente artigo é avaliar a estratégia de utilização de MCs elaborados com o software CmapTools ${ }^{\circledR}$ no curso na modalidade a distância denominado Introdução a Anamnese e ao Exame Físico de Enfermagem. O processo de ensino-aprendizagem foi avaliado sob dois aspectos: a avaliação pelo professor dos MCs produzidos ao longo do curso e a avaliação dos alunos sobre o desenvolvimento da atividade proposta.

A importância da presente investigação reside no fato de que essa estratégia de aprendizagem pode incentivar a participação dos estudantes de enfermagem na construção de conhecimentos em uma perspectiva construtivista e autônoma, além de ser um recurso de informática aplicável tanto na educação a distância, como no ensino presencial de enfermagem. 


\section{MÉTODO}

Este artigo apresenta o estudo de caso de abordagem qualitativa desenvolvido junto a 20 estudantes de enfermagem que realizaram o curso de extensão denominado Introdução a Anamnese e ao Exame Físico de Enfermagem, promovido pela Escola de Enfermagem da Universidade Federal do Rio Grande do Sul (UFRGS), com 60 horas de duração na modalidade a distância entre os meses de abril e junho de 2008.

Os 20 estudantes participantes inscreveram-se no curso voluntariamente, atendendo ao pré-requisito de estarem matriculados na quarta etapa do Curso de Graduação em Enfermagem da mesma universidade.

O curso, desenvolvido no Ambiente Virtual de Aprendizagem (AVA) Moodle ${ }^{\circledR}$, foi dividido em cinco módulos denominados trabalhando no Moodle ${ }^{\circledR}$, referenciais teóricos da anamnese e do exame físico, coleta de informações, técnicas propedêuticas do exame físico e integrando a anamnese e o exame físico.

No módulo 1 - trabalhando no Moodle ${ }^{\circledR}$ - foram desenvolvidas duas oficinas, com uma hora de duração cada, apresentando os recursos do software CmapTools ${ }^{\circledR}$ no Laboratório de Informática da instituição. Aproveitando a proposta de apresentar as ferramentas do ambiente virtual, solicitou-se a leitura de artigo que descrevia a realização de mapas conceituais com estudantes de enfermagem a ser debatido na sala de bate-papo do AVA. ${ }^{2}$

No módulo 2 - referenciais teóricos da anamnese e do exame físico - foi proposta a realização de um MC a partir das idéias referentes ao tema dessa etapa do curso.

A coleta de dados ocorreu durante a realização do curso, por meio da avaliação dos $11 \mathrm{MCs}$ elaborados durante o módulo 2 e a avaliação final do curso realizada por esses, no preenchimento de questionário online e os dados obtidos durante a técnica de grupo focal. Foram realizados seis encontros com essa técnica, mas a avaliação da atividade de desenvolvimento de MCs usando o software CmapTools® ficou evidenciada ao longo desses.

A avaliação dos MCs pela professora ocorreu por meio do preenchimento de formulário elaborado pelas pesquisadoras que contemplava os itens - os conceitos apresentados estão de acordo com o tema em estudo; o mapa demonstra que o estudante compreendeu o tema proposto; o estudante expressa uma reflexão crítica; o mapa contempla a ligação entre diferentes conceitos; os verbos de ligação utilizados expressam a ação entre os conceitos a que se referem; a reflexão expressa vai além do tema em estudo; o design do mapa facilita a sua leitura e interpretação.

Cada um desses itens foi avaliado quanto ao grau de desenvolvimento alcançado em atingiu, em desenvolvimento e não atingiu. $\mathrm{O}$ formulário com as pontuações e os comentários foi disponibilizado no fórum de discussão do Moodle ${ }^{\circledR}$ para conhecimento dos estudantes. Os dados provenientes dos formulários foram analisados e apresentados numericamente.

As avaliações dos estudantes referentes à atividade de realização dos MCs foram selecionadas e processadas com o apoio do software NVivo8®. Em seguida, os dados foram analisados através da técnica da análise temática, adotandose as seguintes etapas: organização do material coletado; construção das categorias descritivas iniciais; desvelamento dos sentidos implícitos e contraditórios; reagrupamento das categorias procurando observar tendências e padrões; e busca de relações e inferências em nível de abstração mais elevado. ${ }^{17}$

Este estudo faz parte do projeto de tese de doutorado intitulado Construção cooperativa do conhecimento em tecnologias do cuidar em enfermagem: anamnese e exame físico em ambiente virtual, que foi aprovado pelo Comitê de Ética em Pesquisa da UFRGS sob No 2007811.

Os estudantes assinaram um Termo de Consentimento Livre e Esclarecido. Portanto, tiveram a sua identidade preservada, sendo assegurado o anonimato, conforme a Resolução No 196/96 do Conselho Nacional deSaúde. Os materiais produzidos pelos participantes foram identificados por nomes fictícios designados pelos próprios sujeitos.

\section{APRESENTAÇÃO E DISCUSSÃO DOS RESULTADOS}

Dos 20 participantes do curso, 18 eram do sexo feminino e dois do masculino. A idade dos estudantes variou entre 20 e 28 anos. Os estudantes nessa faixa etária vivenciaram intensamente a explosão das tecnologias digitais na última década do século XXe, portanto, são denominados nativos digitais. ${ }^{18}$ Isso faz com que esses alunos tenham uma facilidade maior na aprendizagem de novos recursos tecnológicos, pois convivem de longa data com os mesmos, em relação aos mais velhos, que, por sua vez, tiveram que 'imigrar' para as novas formas de comunicação. 
Outra característica do grupo em estudo é que todos os alunos tinham acesso à internet em suas residências, mas essa foi a primeira experiência utilizando um AVA. Em estudo realizado com estudantes de enfermagem da mesma universidade em 2004, identificou-se que $82,9 \%$ desses tinham computador em suas residências, e também que a frequência de utilização do computador estava relacionada ao nível de conhecimento sobre informática que o estudante julgava ter..$^{19}$ Assim constata-se a expansão do acesso aos recursos de informática entre os estudantes de enfermagem, o que faz com que tenham mais habilidades na sua utilização, mas as instituições de ensino pouco exploram ainda as possibilidades da educação a distância.

Cabe salientar que esses estudantes já haviam entrado em contato com MCs em outra disciplina no curso de graduação, mas, na ocasião, eles foram realizados em papel, portanto, esse momento foi o de aprendizagem na utilização do software CmapTools ${ }^{\circledR}$. Duas estudantes não compareceram às oficinas, mas, a distância, foram orientadas quanto à instalação do software, e referiram não ter tido dificuldades em instalá-lo e utilizá-lo.

A apropriação dos conceitos principais necessários para a elaboração de um MC começou a ser apresentada aos estudantes no módulo 1 do curso, quando discutiu-se na sala de bate-papo um texto que apresentava exemplos de mapas conceituais produzidos por estudantes no mesmo nível do curso de graduação que eles. ${ }^{2}$ Os estudantes sinalizaram as principais características constituintes dos MCs como conceitos centrais, verbos de ligação, interligação entre diferentes conceitos, o que os fez expressarem que compor um MC seria uma atividade de difícil execução.

No módulo 2, essa crença foi sendo desfeita quando começaram a praticar nas oficinas a confecção de MCs. A partir do tema referenciais teóricos da anamnese e do exame físico, os estudantes fizeram comentários no fórum de discussões e no bate-papo no Moodle® sobre as suas dúvidas e opiniões sobre o desenvolvimento da sistematização da assistência em enfermagem. $\mathrm{Na}$ finalização desse módulo, foi proposto aos estudantes que elaborassem um MC que poderia ser feito individualmente ou em pequenos grupos e posteriormente publicado no fórum de discussões. Dos 11 MCs elaborados, um foi realizado em trio, quatro individualmente e seis em duplas. Os estudantes que não conseguiram encontrar-se presencialmente para a realização da atividade recorreram a comunicadores instantâneos como o
Windows Live Messenger ${ }^{\circledR}$ e ao correio eletrônico para a elaboração da atividade. A seguir serão apresentadas as avaliações dos mapas conceituais e a avaliação dos alunos sobre o desenvolvimento da atividade.

\section{Avaliação dos mapas conceituais}

A avaliação dos MCs apontou que as 11 produções atingiram plenamente os quesitos referentes à adequação dos conceitos apresentados, à articulação entre diferentes conceitos, além de indicarem que os estudantes haviam compreendido o tema proposto. Assim, conclui-se que os estudantes conseguiram realizar os MCs contemplando as características que os definem, os quais são a apresentação de dois ou mais conceitos conectados por verbos de ligação, para conferir sentido, o que os torna uma proposição. ${ }^{20}$ As proposições são unidades semânticas ou unidades de significado que possibilitam o entendimento das idéias expressas no MC. ${ }^{20}$ Assim, conseguiu-se avaliar nos MCs se os conceitos apresentados estavam adequados e se existem ou não as relações teóricas propostas pelos estudantes.

Existem diferentes tipos de MCs, com distribuições gráficas variadas, sem haver um modelo mais correto ou indicado do que outro. ${ }^{21}$ No presente estudo, não foi proposto nenhum formato pré-estabelecido aos estudantes, e esses ficaram livres para criarem seus MCs. Isso nos remete ao fato de que não existem dois MCs iguais, cada construção é particular, e, se construída coletivamente, será através da negociação de diferentes pontos de vista que o produto final será constituído.

Em contrapartida, ficou evidenciado que a totalidade dos estudantes ainda se encontra em fase de aprendizagem quanto à expressão de uma reflexão crítica. Apenas dois mapas apresentaram conteúdos que não estavam presentes no texto utilizado como ponto de partida para as discussões, sendo que, em um mapa, os estudantes se restringiram a apresentar uma síntese linear do que estava em estudo.

O desenvolvimento da metacognição foi apontado como uma das possibilidades de aplicação dos MCs no ensino de enfermagem, tornando a aprendizagem mais significativa. ${ }^{11}$ Acredita-se que para os estudantes atingirem um nível de abstração que evidencie o pensamento crítico, é necessário um processo mais longo e que os instigue a atingirem tais patamares de construção do conhecimento. 
$\mathrm{Na}$ presente investigação, foi proposta a realização de MCs como uma atividade síntese e integrativa de conceitos que possibilitasse a avaliação dos conhecimentos adquiridos pelo grupo de alunos. O MC como ferramenta avaliativa é apontado como uma estratégia no acompanhamento do processo de ensino-aprendizagem, pois permite que progressivamente as proposições dos alunos sejam observadas e pontuadas. ${ }^{9,20,22}$

$\mathrm{Na}$ estruturação dos MCs, observou-se que 10 deles apresentavam verbos entre conceitos indicando a ação, enquanto um mapa não apresentou verbos de ligação. A colocação de um verbo indicando a ação entre dois ou mais conceitos, como dito anteriormente, é um dos elementos constitutivos das proposições, o que confere significado à unidade.$^{20}$ Esse é $\mathrm{o}$ aspecto que diferencia um MC de um mapa mental, o qual se constitui por ser uma inter-relação entre diferentes conceitos.

$\mathrm{O}$ design de um mapa conceitual facilita a leitura e a interpretação das idéias apresentadas. Nesse quesito, observou-se que 10 mapas conceituais apresentavam uma harmonia entre as formas e as cores, sendo que a distribuição gráfica contemplava as orientações de alfabetização digital mínimas, apesar desse tema não ter sido apresentado aos alunos que intuitivamente desenvolveram um material adequado. Um dos MCs ficou de difícil leitura por ter sido elaborado com letras muito escuras e por ter cor de fundo que não fazia contraste com a cor dessas letras.

\section{Avaliação dos alunos sobre o desenvolvi- mento de mapas conceituais}

O primeiro contato com a possibilidade de realizar um MC com o apoio de um software ocorreu durante o curso. A avaliação dos estudantes sobre a atividade foi bastante positiva, destacando a possibilidade de utilizarem essa técnica em outros contextos como demonstra o seguinte relato: para mim a grande inovação foi fazer meu primeiro mapa conceitual, ferramenta que acredito que na vida profissional vou utilizar bastante. Conhecer o CmapTools ${ }^{\circledR}$ foi muito legal, pois é um programa que podemos utilizar até mesmo para tarefas acadêmicas (Carolina).

Da mesma forma, o MC foi associado à possibilidade de ser utilizado como apoio no desenvolvimento de pesquisas: também tive oportunidade de aprender a trabalhar com os mapas conceituais, que futuramente poderá ser um bom instrumento para organizar planos e projetos (Patrícia).
É interessante observar que a aprendizagem do software CmapTools ${ }^{\circledR}$ não permanece apenas nas suas possibilidades educativas, e que na ótica desses alunos os MCs podem ser utilizados na organização das idéias de diferentes disciplinas e de pesquisas. Os MCs são considerados por estudantes de enfermagem como uma técnica que auxilia na organização dos seus conhecimentos. ${ }^{10}$

Alguns estudantes relataram que o MC foi uma de suas melhores produções ao longo do curso, conforme o relato: considero o mapa conceitual realizado uma participação de destaque. Acredito que ele ficou bem interessante, abrangendo as etapas e muitos detalhes do processo de enfermagem (Anita). A habilidade em conceber um MC aumenta com a prática em fazê-lo, e os estudantes de enfermagem manifestam satisfação com essa estratégia de aprendizagem. $^{12}$

O destaque dado à técnica foi atribuído à possibilidade dos estudantes fazerem sínteses dos temas estudados: acho que todas as minhas participações foram efetivas, mas gostei bastante do meu $M C$, pois procurei relacionar bem os conceitos sobre o processo de enfermagem [...] (Alessandra). A aplicação de MCs no processo de aprendizagem da sistematização da assistência em enfermagem desenvolve a habilidade dos estudantes para estabelecerem inter-relações entre as diferentes etapas e de pensarem criticamente sobre os cuidados de enfermagem. ${ }^{12}$

Um dos objetivos educacionais que o curso procurou desenvolver junto aos estudantes foi o de que esses relacionassem os aprendizados novos com os temas já estudados anteriormente. Esse objetivo foi alcançado conforme demonstra o depoimento: gostei muito de fazer o MC. Fiz com a Patrícia, mas foi bom que discutimos bastante para fazer as ligações e no final acho que deu um resultado bem legal, pois fizemos umas associações bem interessantes com as aulas e com os livros (Isabella).

A proposta construtivista que orientou a realização das atividades de elaboração dos MCs promove a facilitação da aprendizagem e não o ensino conteudista. ${ }^{10,12}$ Os estudantes, ao selecionarem os conceitos e estabelecerem relações entre eles, foram acompanhados pelos professores que os questionaram e desafiaram quanto na problematização dos temas apresentados.

Nos encontros realizados, também ocorreram manifestações avaliativas das atividades de desenvolvimento dos MCs. A troca de pontos de vista entre alunos foi uma das qualidades apontadas quando ocorreu a publicação dos MCs no 
fórum de discussão do ambiente virtual. Na fala da aluna Catarina, observa-se que a mesma sentiu uma mudança durante o curso na maneira como percebia os seus colegas: [...] mas os colegas me surpreenderam bastante, tanto nos chats quanto nos MCs. Antes de vir para cá, eu estava olhando. Eu não fiz o meu, porque eu não fazia nem idéia por onde eu ia começar. O da Alessandra foi um que me chamou bastante atenção, o da Carla ficou bem legal, o do Geferson também. Eu, por exemplo, não sabia que eu concordava em muitas coisas como Geferson, que é uma pessoa com quem eu não converso, acho que nem bom dia a gente se dá. Mas é, mas a gente concorda (Catarina).

A reciprocidade entre colegas é um elemento constitutivo dos processos cooperativos de aprendizagem, e o trabalho em grupos é um facilitador desse ambiente de trocas sociais. ${ }^{23}$ A reflexão gerada pelo acompanhamento dos MCs dos colegas sinaliza o potencial de produção coletiva que essa técnica possui, e, com o apoio do software CmapTools ${ }^{\circledR}$, há uma facilitação na sua elaboração. ${ }^{20}$ Destaca-se que existe a possibilidade de instalação gratuita de uma versão colaborativa desse software, mas no curso não utilizou-se esse recurso.

\section{CONSIDERAÇÕES FINAIS}

Esta investigação teve como objetivo a avaliação de uma estratégia de utilização de MCs elaborados por alunos de enfermagem com o apoio do software CmapTools ${ }^{\circledR}$ em um curso de extensão na modalidade a distância na Escola de Enfermagem da UFRGS.

Pode-se considerar que para o grupo em estudo, ou seja, estudantes do curso de graduação em enfermagem de uma universidade pública, o acesso a internet e a rápida aprendizagem da utilização de recursos de informática no ensino são fatores que motivam iniciativas como a da realização de MCs com o software CmapTools ${ }^{\circledR}$, que é de fácil aprendizagem por ter sua versão em português e existirem vários tutoriais disponíveis na web.

A construção dos MCs, enquanto técnica de ensino, demonstrou ser importante como sinalizador dos conceitos apreendidos pelos estudantes no processo de ensino-aprendizagem, além de integrar o conhecimento novo aos anteriores ao estabelecer inter-relações. Desta forma, é possível pensar-se nos MCs como uma estratégia de acompanhamento da aprendizagem do aluno ao longo de uma disciplina ou de um curso.

$\mathrm{Na}$ elaboração desses, muitas vezes não foi possível promover os espaços necessários de encontro entre os alunos para sua confecção, o que foi solucionado pelos próprios sujeitos da investigação por meio da utilização da comunicação síncrona via comunicadores instantâneos (MSN®) e pelo envio das versões via correio eletrônico. Este fato mais uma vez demonstra a predisposição e o interesse dos alunos de enfermagem em utilizar as tecnologias da comunicação e da informação.

O pensamento crítico é um dos aspectos que deve ser considerado como foco em práticas educativas futuras que desenvolvam MCs, pois constatou-se que os materiais elaborados pelos estudantes deixaram a desejar em termos de aprofundamento e reflexão temática.

Outro aspecto que deve ser futuramente considerado é a utilização da ferramenta de compartilhamento presente no CmapTools ${ }^{\circledR}$. A partir do acesso ao software hospedado em um servidor pode-se disponibilizar a versão editável dos MCs, o que facilita o desenvolvimento do trabalho coletivo entre os estudantes.

Conclui-se que a atividade foi avaliada positivamente pelos sujeitos do estudo e não houve dificuldades na aquisição de conhecimento referentes à tecnologia digital. A proposta que é lançada pelas pesquisadoras é que, da mesma forma que os alunos aceitaram o desafio de conhecer um novo recurso tecnológico, os professores dos cursos de graduação em enfermagem também aceitem esse desafio, sendo agentes multiplicadores dessas tecnologias.

\section{REFERENNCIAS}

1. Clayton LH. Concept mapping: an effective, active teaching-learning method. Nurs Educ Perspect. 2006 Jul-Aug; 27(4):197-203.

2. Abel WM, Freeze M. Evaluation of concept mapping in an associate degree nursing program. J Nurs Educ. 2006 Sept; 45(9):356-64.

3. Ellerman CR, Kataoka-Yashiro MR, Wong LC. Logic models used to enhance critical thinking. J Nurs Educ. 2006 Jun; 45(6):220-7.

4. Founteyn M. Concept mapping: an easy teaching strategy that contributes to understanding and may improve critical thinking. J Nurs Educ. 2007 May; 46(5):199-200.

5. Harpaz I, Balik C, Ehrenfeld M. Concept mapping: an educational strategy for advancing nursing education. Nurs Forum. 2004 Apr-Jun; 39(2):27-30.

6. Hsu LL, Hsieh SI. Concept maps as an assessment tool in a nursing course. J Prof Nurs. 2005 May-Jun; 21(3):141-9. 
7. Wheeler LA, Collins SKR. The influence of concept mapping on critical thinking in baccalaureate nursing students. J Prof Nurs. 2003 Nov-Dec; 19(6):339-46.

8. Akinsanya C, Williams M. Concept mapping for meaningful learning. Nurse Educ Today. 2004 Jan; 24:41-6.

9. MacNeil MS. Concept mapping as a means of course evaluation. J Nurs Educ. 2007 May; 46(5):232-4.

10. Conceição SCO, Taylor LD. Using a constructivist approach with online concept maps: relationship between theory and nursing education. Nurs Educ Perspect. 2007 Sep-Oct; 28(5):268-75.

11. August-Brady MM. The effect of a metacognitive intervention on approach to and self-regulation of learning in baccalaureate nursing students. J Nurs Educ. 2005 Jul; 44(7):297-304.

12. Hinck SM, Webb P, Sims-Giddens S, Helton C, Hope KL, Utley R, Savinske D, Fahey EM, Yarbrough S. Student learning with concept mapping of care plans in community-based education. J Prof Nurs. 2006 Jan-Feb; 22(1):23-9.

13. Giddens J. Concept mapping as a group learning activity in graduate nursing education. J Nurs Educ. 2006 Jan; 45(1):45-6.

14. Kostovich CT, Poradzisz M, Wood K, O’Brian KL. Learning style preference and student aptitude for concept maps. J Nurs Educ. 2007 May; 46(5):225-31.

15. Institute for Human and Machine CognitionCmapTools [página da internet]. University of West
Florida. [acesso 2008 Nov 19] Disponível em: http:/ / cmap.ihmc.us

16. Falcón GS, Erdmann AL, Meirelles BHS. A complexidade na educação dos profissionais para o cuidado em saúde. Texto Contexto Enferm. 2006 Abr-Jun; 15(2):343-51.

17. Lüdke M, André MEDA. Pesquisa em educação: abordagens qualitativas. São Paulo (SP): EPU; 1986.

18. Prensky M. Digital natives, digital immigrants. Horizon. 2001 Oct; 9(5):1-6.

19. Severo CL, Cogo ALP. Acesso e conhecimento de acadêmicos de enfermagem acerca de recursos computacionais. Rev Gaúcha Enferm. 2006 Dez; 27(4):516-23.

20. Novak JD, Cañas AJ. The theory underlying concept maps and how to construct and use them. [página da internet]. Institute for Human and Machine Cognition Institute for Human and Machine Cognition; 2006 [acesso 2008 Nov 21]. Disponível em: http://cmap.ihmc.us/ Publications/ResearchPapers/TheoryCmaps/ TheoryUnderlyingConceptMaps.htm

21. Tavares R. Construindo mapas conceituais. Ciências Cognição. 2007; 12(4):72-85.

22. Hsu L-L. Developing concepts maps from problembased learning scenario discussions. J Adv Nurs. 2004 Dec; 48(5):510-8.

23. Piaget J. Sobre a pedagogia: textos inéditos. São Paulo (SP): Casa do Psicólogo; 1998. 